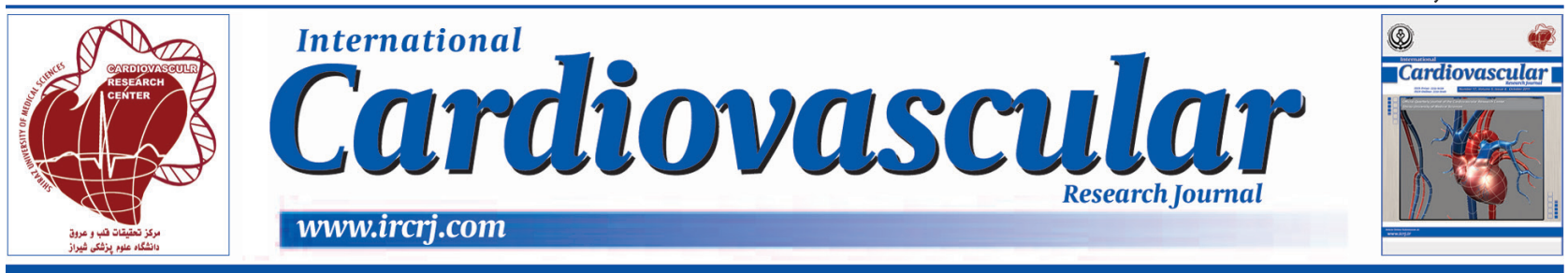

\title{
Role of Gender in the Prevalence of Metabolic Syndrome and Its Related Risk Factors in Shiraz Healthy Heart Center Population
}

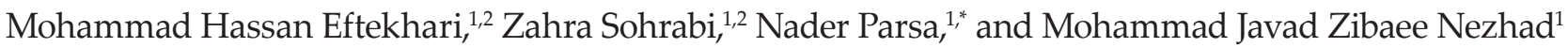

${ }^{1}$ Cardiovascular Research Center, Shiraz University of Medical Sciences, Shiraz, IR Iran

${ }^{2}$ School of Nutrition and Food Sciences, Shiraz University of Medical Sciences, Shiraz, IR Iran

\section{A R T I C L E I N F O}

Article Type:

Research Article

Article History:

Received: 21 Jan 2015

Accepted: 28 Apr 2015

\section{Keywords:}

X-Syndrome

Waist Circumference

Hypertension

Abdominal Obesity

\begin{abstract}
A B S T R A C T
Background: Metabolic Syndrome (MetS) is defined as a group of cardiovascular risk factors and is an important health threat.

Objectives: This study aimed to determine the role of gender in the prevalence of MetS among Shiraz Healthy Heart Center population.

Patients and Methods: This study was performed on 350 adults aged 20 - 65 years from Shiraz Healthy Heart Center, Shiraz University of Medical Sciences. Individuals with obesity secondary to medication and genetic or endocrine disorders were excluded from the study. Weight and height were measured for calculating Body Mass Index (BMI). Waist circumference and blood pressure were measured, as well. Plasma glucose, serum High Density Lipoprotein-cholesterol (HDL-c), and Triglyceride (TG) were also analyzed. The presence of MetS was determined using Adult Treatment Panel-III (ATPIII) criteria.

Results: The results showed significant correlations between age and waist circumference, Fasting Blood Sugar (FBS), Systolic Blood Pressure (SBP), and Diastolic Blood Pressure (DBP). Moreover, statistically significant relationships were found between waist circumference and TG, SBP, DBP, and BMI. Significant correlations were also observed between FBS and TG, SBP, and DBP. Besides, there were strong correlations between TG and HDL-c, SBP, DBP, and BMI. Moreover, BMI, SBP, and DBP were significantly correlated. In addition, serum HDL-c was negatively associated with most of the variables. The prevalence of pre-diabetes, pre-hypertension, and hypertriglyceridemia was higher among males. On the other hand, the prevalence of hypertension, diabetes, high waist circumference, and MetS was significantly higher among the female participants.

Conclusions: A better understanding of the role of gender in the prevalence of MetS is important in developing prevention and treatment strategies.
\end{abstract}

Implication for health policy/practice/research/medical education

Early identification and active management of MetS, differentiating this abnormality and its related risk factors between genders, and assessing their correlations might be necessary to define better strategies for a good management of MetS. A better understanding of the mechanisms about gender differences in MetS might be important in practical interventions toward prevention and treatment of this syndrome. It is noteworthy to declare that the outcome of remedies targeting MetS may depend on age, gender, and some risk factors and their correlations.

\section{Background}

Metabolic syndrome or "X-syndrome" is a group of cardiovascular risk factors including central or abdominal obesity, dyslipidemia, Hypertension (HTN), and insulin

*Corresponding author: Nader Parsa, Cardiovascular Research Center of Shiraz University of Medical Sciences, Research Tower, Khalili Ave. Mollasadra St., Shiraz, Iran, Tel/Fax: +98-7136281562,

E-mail:naderparsa2043@gmail.com resistance (1-4), which are health threats (1). Individuals with MetS are susceptible to Cardiovascular Diseases (CVDs) and type 2 diabetes $(5,6)$. In addition to CVD and diabetes, those with MetS are at a higher risk for polycystic ovarian syndrome, fatty liver, cholesterolemia, gallstones, asthma, sleep disturbances, and some types of cancer. Components of MetS are a cluster of major, underlying, and emerging risk factors. Underlying risk factors for 
CVDs are central or abdominal obesity, sedentary lifestyle, and atherogenic diet. Besides, the major risk factors are HTN, high Low-Density Lipoprotein-cholesterol (LDLcholesterol), low High-Density Lipoprotein-cholesterol (HDL-c), cigarette smoking, premature familial Coronary Heart Disease (CHD), and aging. The emerging risk factors encompass high Triglyceride (TG) levels, small LDL particles, insulin resistance, glucose intolerance, and pro-inflammatory and pro-thrombotic states (1). As to the components of MetS, abdominal obesity is highly correlated to MetS and is defined by increased waist circumference. Another component of MetS, atherogenic dyslipidemia, is demonstrated by high TG and low HDL levels. High blood pressure, which is highly correlated to obesity and is more prevalent in insulin-resistant patients, is also categorized in MetS. Insulin resistance is also a metabolic risk factor that is strongly associated with CVDs. Additionally, a proinflammatory state, which is defined as high C-Reactive Protein (CRP), is usually present in patients with MetS. The main cause of this state is obesity. Finally, a prothrombotic state is correlated to MetS associated with proinflammatory state (1). Metabolic disturbances due to MetS affect more than $30 \%$ of adults in Tehran, Iran (7). This estimated prevalence is higher than that in most developed countries, such as the United States (8). The estimated prevalence of MetS was almost $25 \%$ in individuals over the age of 20 years, and it affected almost $45 \%$ of those over 50 years in industrialized countries (9). Evidence has demonstrated that the prevalence of MetS has reached a threatening level and a defined target for secondary prevention in National Cholesterol Education Program (10). Because of the increasing trend in obesity (11), the prevalence of MetS is rising (11-14).

Considering blood pressure as one of the components of MetS, high Systolic Blood Pressure (SBP) in women is associated with cardiovascular risk (15). Gender differences in CHD and CVD mortality can be partially explained by higher prevalence of HTN in females (16). Puberty in men, compared to women, with later onset can be another reason for lower blood pressure in men (17). Additionally, a progressive increase in the prevalence of HTN can be found with higher adipose tissue or waist circumference as another component of MetS (18). Body Mass Index (BMI) is considered an independent risk factor for HTN in women (18), rooting from steroid hormones and weight in women (19). Studies have found that changes in weight and adipose tissue contents can affect insulin resistance. Elevated blood glucose level, a component of MetS in women, was also found to cause higher vulnerability to diabetes incidence in spite of normal glucose metabolism $(20,21)$. Serum concentrations of HDL-c and TG are other risk factors of MetS. Furthermore, lower TG and higher HDL-c levels are predominantly seen in pre-menopausal women due to the effects of estrogen $(22,23)$.

\section{Objectives}

Understanding differences between genders regarding MetS components might result in better strategies for MetS identification and management. Currently, limited studies have been performed on the prevalence of MetS in different genders in Iran, especially Shiraz (capital of Fars province). Therefore, this study aims to determine the role of gender in the prevalence of MetS and its related risk factors in the individuals referred to Healthy Heart Center of Shiraz University of Medical Sciences, Shiraz, Iran.

\section{Patients and Methods}

In this cross-sectional, experimental study, 350 subjects were selected from Healthy Heart Center of Shiraz, Iran by systematic random sampling. This program is under the supervision of Shiraz University of Medical Sciences that predominantly works on screening programs. People are referred to this center from various organizations, institutes, and public community from different ethnic or occupational groups of Shiraz metropolitan city. The study protocol was approved by the Research Council of the Heart Research Center at the University. All the participants completed written informed consents and a socio-demographic questionnaire containing information about age, occupation, and marital status.

The individuals aging 20 - 65 years were included in the study, while those with obesity secondary to medication and genetic or endocrine disorders were excluded.

BMI was computed for each participant as body weight divided by height squared. Weight was measured using digital scales to the nearest 100 grams while the participants were barefooted and lightly clothed. Height was also recorded by a tape measure in standing position with the shoulders straight from head to heel with bare feet. Waist circumference was measured at the narrowest level between the lower rib and iliac crest and recorded to the approximately 0.1 centimeter. All the measurements were done by the same person to minimize possible error. The individuals with BMI over $30 \mathrm{~kg} / \mathrm{m} 2$ and between 25 and $29.9 \mathrm{~kg} / \mathrm{m} 2$ were considered as obese and overweight, respectively (4).

Blood pressure was measured twice using a standard mercury sphygmomanometer with the participant seated with 30 second intervals between the measurements. The average blood pressure between the two measurements was recorded.

Adult Treatment Panel-III (ATPIII) criteria were used for determining the presence of MetS (1). The participants with three or more of the following criteria were considered to have MetS (Table 1):

For laboratory measurements, blood samples were taken from the participants after an overnight fasting (12hour). Fasting Blood sugar (FBS) was analyzed using the calorimetric method with glucose oxidase. Enzymatic reagents were used for measuring serum TG. Finally, serum HDL-c was recorded after precipitation of apolipoprotein-B containing lipoproteins with phosphotangestic acid.

The SPSS statistical software, version 16 (SPSS Inc., Chicago, IL) was used for statistical analyses. The correlations between dependent and independent variables were defined and assessed. Then, these observations were evaluated at $\alpha$-level of 0.05 . Multivariate analysis of the variables was also applied.

The correlations between the variables were measured using 2-tailed Pearson's correlation. Mean and standard deviation 


\begin{tabular}{llll}
\hline \multicolumn{1}{l}{ Table 1. Criteria for Metabolic Syndrome } & & \\
\hline & Criteria & Male & Female \\
\hline $\mathbf{1}$ & Waist circumference & $>102 \mathrm{~cm}$ & $>88 \mathrm{~cm}$ \\
$\mathbf{2}$ & HDL-c & $<60 \mathrm{mg} / \mathrm{dL}$ & $<40 \mathrm{mg} / \mathrm{dL}$ \\
$\mathbf{3}$ & Serum triglyceride & $\geq 150 \mathrm{mg} / \mathrm{dL}$ & \\
$\mathbf{4}$ & Blood pressure & $\geq 130 / 85 \mathrm{mmHg}$ & \\
$\mathbf{5}$ & Fasting blood sugar & $\geq 110 \mathrm{mg} / \mathrm{dL}$ & \\
\hline
\end{tabular}

of the variables were calculated according to gender. Coevaluation of the variables was also done using MANOVA.

In qualitative analysis of the variables, the prevalence of the abnormalities according to different genders was compared by chi-square test at the significant level of 0.05 . In case valid $\mathrm{P}$ values were not obtained in chi-square test, Fisher's exact test was used.

\section{Results}

Mean and standard deviation of the variables according to gender have been presented in Table 2. Accordingly, most of the variables had significant correlations at the level of 0.05 . The results of t-test demonstrated strongly significant differences between the two genders regarding MetS variables (Table 2).

Descriptive data regarding the variables' grand means based on the $95 \%$ confidence intervals have been shown in Table 3. The estimated marginal means of the study parameters have been presented in Table 4.

The results of Fisher's test showed a significant difference

\begin{tabular}{|c|c|c|c|c|c|c|c|c|c|}
\hline Variable & Test & Age & Waist & FBS & TG & HDL & SBP & DBP & BMI \\
\hline \multirow{2}{*}{ Age } & Pearson correlation & 1 & $0.153^{\mathrm{a}}$ & $0.168^{\mathrm{a}}$ & 0.034 & 0.081 & $0.400^{\mathrm{a}}$ & $0.201^{\mathrm{a}}$ & 0.072 \\
\hline & $P$ value & & 0.004 & $0.002^{\mathrm{a}}$ & 0.523 & 0.128 & $<0.001$ & 0.000 & 0.178 \\
\hline \multirow{2}{*}{ Waist } & Pearson correlation & & 1 & 0.081 & $0.190^{\mathrm{a}}$ & -0.005 & 0.226 & $0.186^{\mathrm{a}}$ & $0.781^{\mathrm{a}}$ \\
\hline & $P$ value & & & 0.127 & $<0.001$ & 0.932 & $<0.001$ & $<0.001$ & $<0.001$ \\
\hline \multirow{2}{*}{ FBS } & Pearson correlation & & & 1 & $0.180^{\mathrm{a}}$ & -0.044 & $0.241^{\mathrm{a}}$ & $0.183^{\mathrm{a}}$ & 0.060 \\
\hline & $P$ value & & & & 0.001 & 0.404 & $<0.001$ & 0.001 & 0.258 \\
\hline \multirow{2}{*}{ TG } & Pearson correlation & & & & 1 & $-0.394^{a}$ & $0.167^{\mathrm{a}}$ & $0.155^{a}$ & $0.129^{\mathrm{a}}$ \\
\hline & $P$ value & & & & & $<0.001$ & 0.002 & 0.004 & 0.015 \\
\hline \multirow{2}{*}{ HDL } & Pearson correlation & & & & & 1 & -0.041 & -0.044 & -0.003 \\
\hline & $P$ value & & & & & & 0.440 & 0.415 & 0.955 \\
\hline \multirow{2}{*}{ SBP } & Pearson correlation & & & & & & 1 & $0.633^{\mathrm{a}}$ & $0.137^{\mathrm{a}}$ \\
\hline & $P$ value & & & & & & & $<0.001$ & 0.010 \\
\hline \multirow{2}{*}{ DBP } & Pearson correlation & & & & & & & 1 & $0.135^{\mathrm{a}}$ \\
\hline & $P$ value & & & & & & & & 0.011 \\
\hline \multirow{2}{*}{ BMI } & Pearson correlation & & & & & & & & 1 \\
\hline & $P$ value & & & & & & & & \\
\hline
\end{tabular}

${ }^{a}$ Correlation is significant at 0.01 level (2-tailed). Correlation is significant at 0.05 level (2-tailed).

Abbreviations: FBS, fasting blood sugar; TG, triglyceride; HDL, high density lipoprotein; SBP, systolic blood pressure; DBP, diastolic blood pressure; BMI, body mass index

\begin{tabular}{|c|c|c|c|c|c|}
\hline Variable & Gender & $\mathbf{N}$ & Mean \pm SD & T2Hostelling & Multivariate (Significance Level) \\
\hline \multirow{2}{*}{ Waist } & Male & 133 & $94.84 \pm 10.98$ & \multirow{12}{*}{13.55} & \multirow{12}{*}{$\mathrm{P}<0.001$} \\
\hline & Female & 217 & $98.18 \pm 12.15$ & & \\
\hline \multirow{2}{*}{ TG } & Male & 133 & $193.38 \pm 87.93$ & & \\
\hline & Female & 217 & $172.22 \pm 82.41$ & & \\
\hline \multirow{2}{*}{ HDL } & Male & 133 & $47.93 \pm 8.98$ & & \\
\hline & Female & 217 & $55.85 \pm 10.90$ & & \\
\hline \multirow{2}{*}{ SBP } & Male & 133 & $126 \pm 15.70$ & & \\
\hline & Female & 217 & $121 \pm 18.55$ & & \\
\hline \multirow{2}{*}{ DBP } & Male & 133 & $78.66 \pm 11.37$ & & \\
\hline & Female & 217 & $73.95 \pm 11.34$ & & \\
\hline \multirow{2}{*}{ BMI } & Male & 133 & $26.59 \pm 3.82$ & & \\
\hline & Female & 217 & $28.67 \pm 5.26$ & & \\
\hline \multirow{2}{*}{ FBS } & Male & 133 & $111.21 \pm 30.73$ & \multirow{2}{*}{$0.003^{+}$} & \multirow{2}{*}{0.960} \\
\hline & Female & 217 & $110.99 \pm 34.40$ & & \\
\hline
\end{tabular}

Abbreviations: FBS, fasting blood sugar; TG, triglyceride; HDL, high density lipoprotein; SBP, systolic blood pressure; DBP, diastolic blood pressure; BMI, body mass index; NS, not significant

${ }^{+}$Independent sample t-test 
Table 4. Estimated Marginal Means (Grand Mean) of the Study Variables

\begin{tabular}{llll}
\hline \multirow{2}{*}{ Variables } & Mean & \multicolumn{2}{c}{ 95\% Confidence Interval } \\
\cline { 3 - 4 } & & Lower bound & Upper bound \\
\hline Waist circumference & 96.52 & 95.25 & 97.79 \\
FBS & 111.12 & 107.53 & 114.71 \\
TG & 182.91 & 173.74 & 192.09 \\
HDL & 51.89 & 50.79 & 53.00 \\
SBP & 123.87 & 121.97 & 125.77 \\
DBP & 76.31 & 75.08 & 77.54 \\
BMI & 27.63 & 27.12 & 28.151 \\
\hline
\end{tabular}

Abbreviations: FBS, fasting blood sugar; TG, triglyceride; HDL, high density lipoprotein; SBP, systolic blood pressure; DBP, diastolic blood pressure; BMI, body mass index

between the two genders concerning various stages of HTN. However, no significant difference was seen between males and females concerning FBS in the normal range (Table 5). Nevertheless, Pearson's chi-square test revealed a significant difference between males and females regarding high FBS levels $(\mathrm{P}=0.031)$.

The study results revealed a significant difference between males and females with respect to high waist circumference (more than the normal levels). Additionally, the prevalence of this abnormality was 3 folds higher among females compared to males ( 78.7 vs. $21.8 \%$; $\mathrm{P}<0.001$ ).

The study findings indicated no significant differences between the two genders with regard to serum TG concentration in normal ranges. However, in the abnormal ranges of serum TG, this difference was clearly significant by Pearson's chi-square test $(\mathrm{P}=0.017)$ (Table 5$)$.

The prevalence of various risk factors was not significantly different between the males and females with 1,2, and 3 risk factors. Also, no significant difference was observed between the male and female participants with 4 risk factors. Although this rate was two times higher in females compared to males (12.4 vs. $6.8 \%$ ), due to the small sample size, this difference was not statistically significant. The prevalence of MetS was also assessed in both genders with 3 or more risk factors (Table 6).

In this study, SBP was evaluated and the results showed a significant difference between males and females concerning the prevalence of prehypertension $(\mathrm{P}=0.021)$ (Table 6).

This study also analyzed the prevalence of various classifications of FBS in both genders. According to the results, the prevalence of pre-diabetes was not different between males and females. Also, the prevalence of diabetes was $8.3 \%$ and $10 \%$ in males and females, respectively and the difference was not statistically significant (Table 5).
In the stratified analysis of serum TG levels based on different TG levels (borderline high, high, and very high), significant differences were seen between the males and females with high serum TG levels $(P=0.022)$ (Table 6). However, no significant difference was observed between those with very high serum TG levels due to the very small sample size.

Finally, the results of Pearson's chi-square test demonstrated a significant difference between the two genders regarding different levels of BMI (overweight and obesity) $(\mathrm{P}=0.002)$ (Table 6). This difference was confirmed by likelihood standardized statistics and linear by linear association.

\section{Discussion}

MetS and its related risk factors have become worldwide threats due to their links to different chronic diseases (9). Findings of the present study indicated significant correlations between age and waist circumference, FBS, SBP, and DBP. Furthermore, statistically significant relationships were found between waist circumference and TG, SBP, DBP, and BMI. Also, significant correlations were demonstrated between FBS and TG, SBP, DBP. There were high correlations between TG and HDL-c, SBP, DBP, and BMI. Additionally, significant correlations exist between BMI and systolic and diastolic blood pressure.

Positive correlations were found between waist circumference and systolic and diastolic blood pressure. In a study by Yalcin et al. correlations between adiposity and blood pressure was detected (18) which supported our results regarding the correlations between waist circumference and blood pressure. The results of the present study considering the significant correlations between age and MetS risk factors are supported by Arthur et.al study (24). A negative correlation was found

\begin{tabular}{|c|c|c|c|c|}
\hline \multirow[t]{2}{*}{ Variables } & & \multicolumn{2}{|c|}{ Gender } & \multirow[t]{2}{*}{ P value } \\
\hline & & Male & Female & \\
\hline \multirow[t]{2}{*}{ High waist circumference } & Yes & $21.8 \%(29)$ & $78.8 \%(171)$ & 0.001 \\
\hline & No & $78.2 \%(104)$ & $21.2 \%(46)$ & \\
\hline \multirow[t]{2}{*}{ High FBS } & Yes & $67.7 \%$ ( 90$)$ & $55.8 \%(124)$ & 0.031 \\
\hline & No & $32.3 \%(43)$ & $44.2 \%(96)$ & \\
\hline \multirow[t]{2}{*}{ High TG } & Yes & $62.4 \%(83)$ & $49.3 \%(107)$ & 0.017 \\
\hline & No & $37.6 \%(50)$ & $50.7 \%(110)$ & \\
\hline \multirow[t]{2}{*}{ HTN } & Yes & $45.1 \%(60)$ & $32.7 \%(71)$ & 0.015 \\
\hline & No & $54.9 \%(73)$ & $67.3 \%(146)$ & \\
\hline
\end{tabular}

Abbreviations: NS, not significant; FBS, fasting blood sugar; TG, triglyceride; HTN, hypertension 


\begin{tabular}{|c|c|c|c|c|}
\hline \multirow[t]{2}{*}{ Risk Factors } & & \multicolumn{2}{|c|}{ Gender } & \multirow[t]{2}{*}{ P value } \\
\hline & & Male & Female & \\
\hline Normal & No risk factors & $10(7.5 \%)$ & $17(7.8 \%)$ & 0.481 \\
\hline \multirow[t]{4}{*}{ Different risk factors } & Yes, 1- Risk factor & $34(25.6 \%)$ & $46(21.1 \%)$ & \\
\hline & Yes, 2- Risk factors & $45(33.8 \%)$ & $68(31.2 \%)$ & \\
\hline & Yes, 3- Risk factors & $35(26.3 \%)$ & $60(27.5 \%)$ & \\
\hline & Yes, 4- Risk factors & $9(6.8 \%)$ & $27(12.4 \%)$ & \\
\hline \multirow[t]{2}{*}{ Total risk factors } & Yes & $45(33.1 \%)$ & $87(39.9 \%)$ & 0.200 \\
\hline & No & $89(66.9 \%)$ & $131(60.1 \%)$ & \\
\hline \multirow[t]{4}{*}{ SBP } & Normal & $45(34.6 \%)$ & $112(50.9 \%)$ & 0.021 \\
\hline & Pre-hypertension & $64(49.2 \%)$ & $76(34.5 \%)$ & \\
\hline & Stage- 1 hypertension & $55(11.5 \%)$ & $25(11.4 \%)$ & \\
\hline & Stage-2 hypertension & $6(4.6 \%)$ & $7(3.2 \%)$ & \\
\hline \multirow[t]{3}{*}{ FBS } & Normal & $38(28.6 \%)$ & $88(39.8 \%)$ & 0.057 \\
\hline & Pre-diabetic & $84(63.2 \%)$ & $111(50.2 \%)$ & \\
\hline & Diabetic & $11(8.3 \%)$ & $22(10.0 \%))$ & \\
\hline \multirow[t]{4}{*}{ TG } & Normal & $50(37.6 \%)$ & $112(50.7 \%)$ & 0.022 \\
\hline & Borderline high & $27(20.3 \%)$ & $48(21.7 \%)$ & \\
\hline & High & $55(41.4 \%)$ & $61(27.6 \%)$ & \\
\hline & Very high & $1(0.8 \%)$ & $0(0.0 \%)$ & \\
\hline \multirow[t]{4}{*}{ BMI } & Underweight & $2(1.5 \%)$ & $4(1.9 \%)$ & 0.002 \\
\hline & Normal weight & $47(35.3 \%)$ & $51(23.7 \%)$ & \\
\hline & Overweight & $59(44.4 \%)$ & $79(36.7 \%)$ & \\
\hline & Obesity & $25(18.8 \%)$ & $81(37.7 \%)$ & \\
\hline
\end{tabular}

Abbreviations: SBP, systolic blood pressure; FBS, fasting blood sugar; TG: triglyceride; BMI, body mass index

between serum TG and HDL-cholesterol in the current study and this finding was seen in previous studies as well $(23,25)$. Biologically, the balance between HDL-c and TG is mediated through an enzyme called cholesterol ester transfer protein (CETP). Additionally, transfer of TG from TG-rich lipoproteins to HDL-c is done using this enzyme in exchange for cholesteryl esters, and that's why a negative correlation between these two variables can be found according to CETP activity (26). HDL-c and TG are considered important risk factors for CVD (27, 28) and these correlations can be important. Moreover, BMI and waist circumference are considered the markers of adiposity. The present study finding according to correlations between blood pressure and body mass index was supported by previous studies related to the fact that BMI and adiposity are strong independent determinants for blood pressure and the effects of central obesity (android obesity) on blood pressure (18). However, in another study, despite an increasing trend in abdominal obesity, decreasing trends were seen in HDL-c levels (29), The results of our study related to negative correlations between HDL-c and abdominal obesity according to body mass index and waist circumference are consistent with what was mentioned in that study (29).

In the present study HDL-c and other variables (including waist circumference, SBP, DBP, TG, BMI, and FBS) were negatively correlated. The results of the current study are supported by a study in adults, regarding a decrease in HDL-c correlated with an increase in abdominal obesity (29).

By multivariate analysis, almost all of the variables were significantly correlated. A higher prevalence of hypertension was seen in male participants and this was significantly different between genders. This result can be partially explained by a higher prevalence of overweight in males. This finding was supported by another study that men were more likely to develop hypertension in adulthood. Some investigators believe that a higher prevalence of hypertension is reported in females due to their steroidal hormone impacts on blood pressure (19). However, those findings were not supported in the current study.

According to the FBS measurements, more females had diabetes. However, prevalence of pre-diabetes as "impaired fasting glucose" was more among males. Findings of previous studies indicated that changes in weight and adipose tissue can affect insulin resistance and blood sugar in women, as a component of MetS. Therefore, this makes them more susceptible to diabetes (21). Findings of the current study might be partially explained by higher prevalence of obesity and high waist circumference in female participants suffering from diabetes.

In this study significant higher waist circumferences were found among females compared with males. In contrast to the present study results, previous research by Miyatake and his colleagues showed a higher abdominal obesity in males based upon waist circumference (30), which might be due to the differences between the sample size, age, and other related risk factors.

A higher prevalence of hypertriglyceridemia was found among males compared with females, which was statistically different. In another study, lower triglyceride levels in premenopausal women due to the effects of estrogen is in line with the results of the present study in this regard (23).

After analyzing numerous risk factors, more male participants had one or two risk factors in comparison with the females. On the other hand, more women had three or four risk factors in comparison with men, which means that the female participants had a higher prevalence of 
MetS (presence of 3 or more risk factors) according to the findings of this study. A higher prevalence of MetS among women compared with men is supported by some other researches $(21,31)$. Furthermore, in some other studies such as one by Santos et.al, almost the same prevalence in both genders was reported (32). In the current study higher prevalence of MetS in the females can be partially described by the higher prevalence of obesity and higher waist circumference in this group. The results of this study were supported by previous studies that demonstrated the effects of obesity on MetS prevalence $(33,34)$. In general, findings of this study indicated that there are gender differences in the prevalence of metabolic syndrome and its related risk factors.

The limitations of the current study might be the smaller sample size of the males and lack of sufficient data regarding dietary information from study subjects. In addition, female predominance in the participants compared with males in this study might affect the results interpretation.

Strength of this study is the assessment of MetS and its related risk factors based on different genders in a multicenter study population. Moreover, we evaluated all correlations and multivariate analysis of the MetS risk factors that was done almost for the first time in Shiraz population. Further strength of the study is that the participants mostly come from different ethnic and occupational groups from various areas.

In conclusion, women in this study were found to have higher risk for MetS. Significant correlation between abdominal obesity and MetS is implicated especially in women (While it is noteworthy to state that as a normal physiologic phenomenon, higher waist circumferences are reported in postmenopausal women due to estrogen changes). Early identification and active management of MetS and differentiating this abnormality and its related risk factors between genders and assessing their correlations might be necessary to define better strategies for an appropriate management of MetS. A better understanding of the mechanisms about gender differences in MetS might be important in practical interventions toward prevention and treatment of this syndrome. It is noteworthy to express that the outcome of remedies targeting MetS may depend on age, gender and some risk factors of patients and their correlations.

\section{Acknowledgements}

We would like to thank Mehrab Sayadi for assisting us in analyzing the data.

\section{Authors' Contribution}

Study concept and design: Mohammad Hasan Eftekhari, Zahra Sohrabi, Nader Parsa; Acquisition of data: Zahra Sohrabi; Analysis and interpretation of data: Nader Parsa, Zahra Sohrabi; Drafting of the manuscript: Nader Parsa, Zahra Sohrabi, Mohammad Hasan Eftekhari; Critical revision of the manuscript for important intellectual content: Nader Parsa, Zahra Sohrabi, Mohammad Hasan Eftekhari; Statistical analysis: Nader Parsa, Zahra Sohrabi; Administrative, technical, and material support: Mohammad Javad Zibaee Nezhad, Nader Parsa; Study supervision: Mohammad Hasan Eftekhari, Zahra Sohrabi, Nader Parsa

\section{Financial disclosure}

There is no financial disclosure.

\section{Funding/Support}

Shiraz University of Medical Sciences, Cardiovascular Research Center.

\section{References}

1. Grundy SM, Brewer HB, Jr., Cleeman JI, Smith SC, Jr., Lenfant C. Definition of metabolic syndrome: Report of the National Heart, Lung, and Blood Institute/American Heart Association conference on scientific issues related to definition. Circulation. 2004;109(3):433-8.

2. Moebus S, Balijepalli C, Losch C, Gores L, von Stritzky B, Bramlage $\mathrm{P}$, et al. Age- and sex-specific prevalence and ten-year risk for cardiovascular disease of all 16 risk factor combinations of the metabolic syndrome - A cross-sectional study. Cardiovasc Diabetol. 2010;9:34.

3. Pedroza-Tobias A, Trejo-Valdivia B, Sanchez-Romero LM, Barquera S. Classification of metabolic syndrome according to lipid alterations: analysis from the Mexican National Health and Nutrition Survey 2006. BMC Public Health. 2014;14:1056.

4. Yoo S, Nicklas T, Baranowski T, Zakeri IF, Yang SJ, Srinivasan $\mathrm{SR}$, et al. Comparison of dietary intakes associated with metabolic syndrome risk factors in young adults: the Bogalusa Heart Study. Am J Clin Nutr. 2004;80(4):841-8.

5. Lakka HM, Laaksonen DE, Lakka TA, Niskanen LK, Kumpusalo E, Tuomilehto J, et al. The metabolic syndrome and total and cardiovascular disease mortality in middle-aged men. JAMA. 2002;288(21):2709-16.

6. Isomaa B, Almgren P, Tuomi T, Forsen B, Lahti K, Nissen M, et al. Cardiovascular morbidity and mortality associated with the metabolic syndrome. Diabetes Care. 2001;24(4):683-9.

7. Azizi F, Rahmani M, Ghanbarian A, Emami H, Salehi P, Mirmiran $\mathrm{P}$, et al. Serum lipid levels in an Iranian adults population: Tehran Lipid and Glucose Study. Eur J Epidemiol. 2003;18(4):311-9.

8. Ford ES, Giles WH, Dietz WH. Prevalence of the metabolic syndrome among US adults: findings from the third National Health and Nutrition Examination Survey. JAMA. 2002;287(3):356-9.

9. Giovannucci E. Metabolic syndrome, hyperinsulinemia, and colon cancer: a review. Am J Clin Nutr. 2007;86(3):s836-42.

10. Young-Hyman D, Schlundt DG, Herman L, De Luca F, Counts D. Evaluation of the insulin resistance syndrome in 5- to 10-yearold overweight/obese African-American children. Diabetes Care. 2001;24(8):1359-64.

11. Flegal KM, Carroll MD, Ogden CL, Johnson CL. Prevalence and trends in obesity among US adults, 1999-2000. JAMA. 2002;288(14):1723-7.

12. The metabolic syndrome: mechanisms, epidemiology, and clinical implications. Postgrad Med. 2003;114(6 Suppl Managing Metabolic):17-27.

13. Azimi-Nezhad M, Herbeth B, Siest G, Dade S, Ndiaye NC, Esmaily $\mathrm{H}$, et al. High prevalence of metabolic syndrome in Iran in comparison with France: what are the components that explain this? Metab Syndr Relat Disord. 2012;10(3):181-8.

14. Freedman DS, Khan LK, Serdula MK, Galuska DA, Dietz WH. Trends and correlates of class 3 obesity in the United States from 1990 through 2000. JAMA. 2002;288(14):1758-61.

15. Benetos A, Thomas F, Safar ME, Bean KE, Guize L. Should diastolic and systolic blood pressure be considered for cardiovascular risk evaluation: a study in middle-aged men and women. $\mathrm{J}$ Am Coll Cardiol. 2001;37(1):163-8.

16. Benetos A, Rudnichi A, Thomas F, Safar M, Guize L. Influence of heart rate on mortality in a French population: role of age, gender, and blood pressure. Hypertension. 1999;33(1):44-52.

17. Hardy R, Kuh D, Whincup PH, Wadsworth ME. Age at puberty and adult blood pressure and body size in a British birth cohort study. J Hypertens. 2006;24(1):59-66.

18. Yalcin BM, Sahin EM, Yalcin E. Which anthropometric measurements is most closely related to elevated blood pressure? Fam Pract. 2005;22(5):541-7. 
19. Dasgupta K, O’Loughlin J, Chen S, Karp I, Paradis G, Tremblay J, et $a l$. Emergence of sex differences in prevalence of high systolic blood pressure: analysis of a longitudinal adolescent cohort. Circulation. 2006;114(24):2663-70.

20. Onat A, Hergenc G, Keles I, Dogan Y, Turkmen S, Sansoy V. Sex difference in development of diabetes and cardiovascular disease on the way from obesity and metabolic syndrome. Metabolism. 2005;54(6):800-8.

21. Zabetian A, Hadaegh F, Sarbakhsh P, Azizi F. Weight change and incident metabolic syndrome in Iranian men and women; a 3 year follow-up study. BMC Public Health. 2009;9:138.

22. Lafortuna CL, Agosti F, De Col A, Pera F, Adorni F, Sartorio A. Prevalence of the metabolic syndrome and its components among obese men and women in Italy. Obes Facts. 2012;5(1):127-37.

23. Williams CM. Lipid metabolism in women. Proc Nutr Soc. 2004;63(1):153-60.

24. Arthur FK, Adu-Frimpong M, Osei-Yeboah J, Mensah FO, Owusu L. The prevalence of metabolic syndrome and its predominant components among pre-and postmenopausal Ghanaian women. BMC Res Notes. 2013;6:446.

25. Chen W, Srinivasan SR, Elkasabany A, Berenson GS. Cardiovascular risk factors clustering features of insulin resistance syndrome (Syndrome X) in a biracial (Black-White) population of children, adolescents, and young adults: the Bogalusa Heart Study. Am J Epidemiol. 1999;150(7):667-74.

26. Sandhofer A, Kaser S, Ritsch A, Laimer M, Engl J, Paulweber B, et al. Cholesteryl ester transfer protein in metabolic syndrome.
Obesity (Silver Spring). 2006;14(5):812-8.

27. Jeppesen J, Hein HO, Suadicani P, Gyntelberg F. Relation of high TG-low HDL cholesterol and LDL cholesterol to the incidence of ischemic heart disease. An 8-year follow-up in the Copenhagen Male Study. Arterioscler Thromb Vasc Biol. 1997;17(6):1114-20.

28. Mazza A, Tikhonoff V, Schiavon L, Casiglia E. Triglycerides + high-density-lipoprotein-cholesterol dyslipidaemia, a coronary risk factor in elderly women: the CArdiovascular STudy in the ELderly. Intern Med J. 2005;35(10):604-10.

29. Bozorgmanesh MR, Hadaegh F, Padyab M, Mehrabi Y, Azizi F. Temporal changes in anthropometric parameters and lipid profile according to body mass index among an adult Iranian urban population. Ann Nutr Metab. 2008;53(1):13-22.

30. Miyatake N, Wada J, Matsumoto S, Nishikawa H, Makino H, Numata T. Re-evaluation of waist circumference in metabolic syndrome: a comparison between Japanese men and women. Acta Med Okayama. 2007;61(3):167-9.

31. Onat A, Uyarel H, Hergenc G, Karabulut A, Albayrak S, Can G. Determinants and definition of abdominal obesity as related to risk of diabetes, metabolic syndrome and coronary disease in Turkish men: a prospective cohort study. Atherosclerosis. 2007;191(1):182-90.

32. Santos AC, Lopes C, Barros H. Prevalence of metabolic syndrome in the city of Porto. Rev Port Cardiol. 2004;23(1):45-52.

33. Hutley L, Prins JB. Fat as an endocrine organ: relationship to the metabolic syndrome. Am J Med Sci. 2005;330(6):280-9.

34. Waki H, Tontonoz P. Endocrine functions of adipose tissue. Annu Rev Pathol. 2007;2:31-56. 